\title{
Examination of the Classroom Management Profiles of Secondary and Primary School Teachers
}

\author{
Malik Beyleroglu (Associate Professor) \\ Sakarya University Sport Sciences Faculty, Sakarya, Turkey \\ Sakir Bezcu (Assistant Professor) \\ Karabük University Hasan Doğan BESYO, Karabuk, Turkey \\ Muhsin Hazar (Associate Professor) \\ Emre Ozan Tingaz (Research Assistant) \\ Hacer Ozge Baydar (Research Assistant) \\ Gazi University Sport Sciences Faculty, Ankara, Turkey
}

Doi: 10.19044/esj.2017.v13n28p66 URL:http://dx.doi.org/10.19044/esj.2017.v13n28p66

\begin{abstract}
The aim of this study is to examine the Classroom management profiles of secondary education teachers, including physical education and sport teachers, and elementary teachers.

By using a purposeful sampling method in various regions in Turkey, primary school teachers $(n=81)$ and secondary school teachers $(n=100)$ working in Ankara and Gaziantep province constitute the study group. Classroom Management Profile Inventory was used as the operational data collection tool.

There was no statistically significant difference between the scores obtained from the general and sub-dimensions of the classroom management profile inventory when there was a comparison between primary school teachers and secondary school teachers. Neither the secondary school nor elementary teachers' scores on the sub-dimensions and sums of the classroom management profile inventory differ according to branch, age, and sex. However, it was found that the primary school teachers differ in the laissezfaire classroom management profiles according to the placement year. In this sub-dimension, the lowest score belongs to the group "4 years and below", while the highest score belongs to the group "5-7 years". Besides, there was no statistically significant difference on secondary school teachers' scores on the sub-dimensions and sums of the classroom management profile inventory according to placement year.
\end{abstract}

Keywords: Classroom Management Profiles, Primary School Teachers, Secondary School Teachers 


\section{Introduction}

Classroom management can be defined as the management of class activities together with the students for the benefits of the students so as to ensure an effective learning process (Balay, 2012). Classroom management has become one of the most talked about topics in education. Inefficient classroom management also negatively affects the performance of teachers in other areas such as lesson planning, classroom activities, and transfer of information to students. As a result, classroom management has become a crucial issue to a teacher (Yisrael, 2012). Making plans about practices is an important point for a successful classroom management. Consequently, teachers have to take account undesirable behaviors while determining their behavior in this process. In this direction, it will facilitate the task of identifying a flexible and appropriate teaching method (Terzi, 2002).

Teachers have various roles to play in a class. One of the most important of these roles is the management of the class. Effective teaching and learning cannot take place in poor classroom management. If students become irregular and disrespectful, and no specific rules and procedures lead to behavior, chaos becomes the norm. In these situations, both the teacher and the student suffer. Teachers struggle to teach, and learners probably learn much lesser than they should. On the contrary, well-managed classes provide a conducive environment for learning and the development of the teacher. Creating good classroom management requires a lot of effort in teaching the person who is responsible for creating it (Marzano, Marzano \& Pickering, 2003). There is no single best way of classroom management, and you cannot address the situations and the diverse challenges teachers face in a single model or theory (Hue \& Li, 2008). Therefore, teachers' classroom management profiles are listed below.

The authoritarian teacher places firm limits and controls on the students. Here, students are often assigned seats for the entire term. The desks are usually in straight rows and there are no deviations. Students must remain in their seats from the beginning of class and throughout the period. This teacher rarely gives hall passes or recognizes excused absences. Oftentimes, the classroom is very quiet. Students have the awareness that they should not interrupt the teacher. Since verbal exchange and discussion are discouraged, the authoritarian's teachers do not have the opportunity to learn and/or practice communication skills. This teacher prefers vigorous discipline and expects swift obedience. Failure to obey the teacher usually results in detention or a trip to the principal's office. In this classroom, students need to follow directions and not ask why (Dunbar, 2004).

Authoritative Teachers, as the authority figure in the classroom, need to be authoritative rather than either authoritarian or laissez-faire. Teachers have the right and the responsibility to exert leadership and to exercise 
control. Nevertheless, they increase their chances of success if they are understanding and supportive of students, and if they ensure that students understand the reasons behind their demands. However, focusing on desired behavior (stressing what to do rather than what not to do) and following up with cues and reminders is also effective. Teachers should be prepared to supply objectively good reasons for their behavior demands (Brophy, 1996).

The indifferent teacher is not very involved in the classroom. This teacher places few demands, if any, on the students and appears generally uninterested. The indifferent teacher just doesn't want to impose on the students and often feels that class preparation is not worth the effort. Things like field trips and special projects are out of the question. This teacher simply won't take the necessary preparation time and may use the same materials, year after year. Also, classroom discipline is lacking. This teacher may lack the skills, confidence, or courage to discipline students (Dunbar, 2004).

The laissez-faire teacher places few demand or controls on the students. "Do your own thing" describes this classroom. Also, this teacher accepts the students' impulses and actions and is less likely to monitor their behavior. The teacher strives not to hurt the students' feelings and has difficulty saying no or enforcing rules. If a student disrupts the class, the teacher may assume that the student is not getting enough attention. When a student interrupts a lecture, the teacher accepts the interruption with the belief that the student must surely have something valuable to add. When discipline is carried out, it is likely to be inconsistent (Dunbar, 2004).

\section{Method}

The research was conducted in a relational screening model. Relational search models are research models that aim to determine the presence and/or extent of change between two or more variables (Karasar, 2014). The aim of the study is to examine the class management profiles of secondary and Primary school teachers in terms of age, sex and placement year, which are also included in physical education teachers.

\section{Study Group}

The following schools, determined by purposeful sampling, constitutes the study group: Gaziantep Emine Ulusoy Elementary School, Şahinbey Kaplan Brothers Imam Hatip Secondary School, Gaziantep / Şehitkamil - Gaziantep Chamber of Commerce Vocational and Technical Anatolian High School, Şehit Veysel Gündoğdu Anatolian High School, Kdz. Ereğli Gülüç Private Education and Business School, Ankara, Ankara Ahi Evran Vocational and Technical Anatolian High School, Ankara Bilge Kagan Primary School. 


\section{Classroom Management Profile Inventory}

Classroom Management Profit Inventory, which was developed by Kris (1996) and adapted to Turkish by Ekici (2004), has been used as the operational data collection tool. This inventory has four (4) sub dimensions; Authoritative, Authoritarian, Indifferent, Laissez faire. The inventory, organized as a total of 12 items for four class management profile types, allows for personal evaluation. Additionally, there are a total of three items for each classroom management profile. Respondents can value between 1 and 5 on each item. Accordingly, the highest score that respondents may receive from each classroom management profile group is 15 , and the lowest score is 1 . Furthermore, 5 likert type scale held as inventory items were rated as: I totally agree (5 points), I agree (4 points), Hesitant (3 points), disagree (2 points), I definitely disagree (1 point) (Ekici, 2004).

\section{Analysis of Data}

MannWhitney $\mathrm{U}$ and Kruskal-Wallis $\mathrm{H}$ tests were used in the analysis of the data. Independent Samples T-Test and One Way ANOVA were also used in the analysis of the data. Here, the assumptions of normality and homogeneity of variances were superseded. One way ANOVA also used LSD tests from posthoc test statistics to determine the significant difference between the groups. The significance level of the statistical analysis used in the study was accepted as 0.05 . "SPSS 21.0 for Windows" package program was used for the statistical analysis of the data obtained.

\section{Findings}

Table 1. Branches of Secondary School Teachers

\begin{tabular}{|c|c|c|}
\hline & Frequency & Percent $(\%)$ \\
\hline Math Teachers & 20 & 11,0 \\
\hline Turkish Teachers & 20 & 11,0 \\
\hline $\begin{array}{c}\text { Physical Education and Sport } \\
\text { Teachers }\end{array}$ & 20 & 11,0 \\
\hline English Teachers & 20 & 11,0 \\
\hline $\begin{array}{c}\text { Religious Culture and Ethics } \\
\text { Teachers }\end{array}$ & 20 & 11,0 \\
\hline Total & 100 & 100,0 \\
\hline
\end{tabular}

Table 2. Placement Years of Secondary School Teachers

\begin{tabular}{|c|c|c|}
\hline & Frequency & Percent (\%) \\
\hline 1 year and under & 15 & 15,0 \\
\hline 2-4 years & 40 & 40,0 \\
\hline 5-7 years & 11 & 11,0 \\
\hline 8-10 years & 7 & 7,0 \\
\hline 11 years and over & 27 & 27,0 \\
\hline Total & 100 & 100,0 \\
\hline
\end{tabular}


Table 3. Distribution of Secondary School Teachers by Sex

\begin{tabular}{|c|c|c|}
\hline & Frequency & Percent (\%) \\
\hline Women & 43 & 43,0 \\
\hline Men & 57 & 57,0 \\
\hline Total & 100 & 100,0 \\
\hline
\end{tabular}

Table 4. Distribution of Secondary School Teachers by Age

\begin{tabular}{|c|c|c|}
\hline & Frequency & Percent (\%) \\
\hline 24 years and under & 18 & 18,0 \\
\hline 25-29 years & 30 & 30,0 \\
\hline 30-34 years & 24 & 24,0 \\
\hline 35-39 years & 17 & 17,0 \\
\hline 40 years and over & 11 & 11,0 \\
\hline Total & 100 & 100,0 \\
\hline
\end{tabular}

Table 5. Is there a difference in the secondary school teachers' sum of scale and subscales scores by age?

\begin{tabular}{|c|c|c|c|c|c|c|}
\hline & Age & $\mathrm{n}$ & $\begin{array}{c}\text { Order } \\
\text { Average }\end{array}$ & $\begin{array}{c}\text { Degree } \\
\text { of } \\
\text { Freedom }\end{array}$ & $\chi^{2}$ & $\mathrm{P}$ \\
\hline \multirow{5}{*}{$\begin{array}{l}\text { Authoritative } \\
\text { Classroom } \\
\text { management } \\
\text { profile }\end{array}$} & 24 years and under & 18 & 46,00 & \multirow[t]{5}{*}{4} & \multirow[t]{5}{*}{7,584} & \multirow[t]{5}{*}{0,108} \\
\hline & 25-29 years & 30 & 56,73 & & & \\
\hline & 30-34 years & 24 & 57,50 & & & \\
\hline & $35-39$ years & 17 & 36,47 & & & \\
\hline & 40 years and over & 11 & 47,27 & & & \\
\hline \multirow{5}{*}{$\begin{array}{l}\text { Authoritarian } \\
\text { Classroom } \\
\text { management } \\
\text { profile }\end{array}$} & 24 years and under & 18 & 42,81 & \multirow[t]{5}{*}{4} & \multirow[t]{5}{*}{4,690} & \multirow[t]{5}{*}{0,321} \\
\hline & 25-29 years & 30 & 50,63 & & & \\
\hline & 30-34 years & 24 & 48,29 & & & \\
\hline & $35-39$ years & 17 & 62,85 & & & \\
\hline & 40 years and over & 11 & 48,45 & & & \\
\hline \multirow{5}{*}{$\begin{array}{l}\text { Laissez-faire } \\
\text { Classroom } \\
\text { management } \\
\text { profile }\end{array}$} & 24 years and under & 18 & 52,31 & \multirow[t]{5}{*}{4} & \multirow[t]{5}{*}{2,364} & \multirow[t]{5}{*}{0,669} \\
\hline & $25-29$ years & 30 & 46,48 & & & \\
\hline & 30-34 years & 24 & 51,56 & & & \\
\hline & 35-39 years & 17 & 58,00 & & & \\
\hline & 40 years and over & 11 & 44,59 & & & \\
\hline \multirow{5}{*}{$\begin{array}{l}\text { Indifferent } \\
\text { Classroom } \\
\text { management } \\
\text { profile }\end{array}$} & 24 years and under & 18 & 50,50 & \multirow[t]{5}{*}{4} & \multirow[t]{5}{*}{6,045} & \multirow[t]{5}{*}{0,196} \\
\hline & 25-29 years & 30 & 51,45 & & & \\
\hline & 30-34 years & 24 & 49,65 & & & \\
\hline & $35-39$ years & 17 & 60,76 & & & \\
\hline & 40 years and over & 11 & 33,91 & & & \\
\hline \multirow{5}{*}{ Total (Scale) } & 24 years and under & 18 & 42,08 & \multirow[t]{5}{*}{4} & \multirow[t]{5}{*}{6,785} & \multirow[t]{5}{*}{0,148} \\
\hline & $25-29$ years & 30 & 53,18 & & & \\
\hline & 30-34 years & 24 & 53,77 & & & \\
\hline & 35-39 years & 17 & 59,71 & & & \\
\hline & 40 years and over & 11 & 35,59 & & & \\
\hline
\end{tabular}

From Table 5 above, there is no statistically significant difference in the total scores from the subscale of the Authoritarian Classroom 
Management Profile by Age $\left(\chi^{2}(s d=4, n=100)=7,584 ; p=0,108>0,05\right.$. There is no statistically significant difference between the total scores from the subscale of Authoritarian Classroom Management Profit by Age $\left(\chi^{2}(s d=4\right.$, $\mathrm{n}=100)=4,690 ; \mathrm{p}=0,321>0,05$. There is no statistically significant difference between the total scores from the subscale of Laissez-faire Classroom Management Profession by Age $\left(\chi^{2}(s d=4, n=100)=2,364 ; p=0,669>0,05\right.$. There is no statistically significant difference between the total scores from the subscale of Indifferent Classroom Management Profession by Age $\left(\chi^{2}(\mathrm{sd}=4, \mathrm{n}=100)=6,045 ; \mathrm{p}=0,196>0,05\right.$. There is no statistically significant difference between the total scores of the total (general scale) according to age $\left(\chi^{2}(\mathrm{sd}=4, \mathrm{n}=100)=6,785 ; \mathrm{p}=0,148>0,05\right.$.

Table 6 . Is there a difference in the secondary school teachers' sum of scale and subscales scores by placement year?

\begin{tabular}{|c|c|c|c|c|c|c|}
\hline & Placement Year & $\mathrm{n}$ & $\begin{array}{c}\text { Order } \\
\text { Average }\end{array}$ & $\begin{array}{c}\text { Degree of } \\
\text { Freedom }\end{array}$ & $\chi^{2}$ & $\mathrm{P}$ \\
\hline \multirow{5}{*}{$\begin{array}{l}\text { Authoritative } \\
\text { Classroom } \\
\text { management } \\
\text { profile }\end{array}$} & 1 year and under & 15 & 45,53 & \multirow[t]{5}{*}{4} & \multirow[t]{5}{*}{3,088} & \multirow[t]{5}{*}{0,543} \\
\hline & 2-4 years & 40 & 49,28 & & & \\
\hline & 5-7 years & 11 & 48,91 & & & \\
\hline & $8-10$ years & 7 & 41,71 & & & \\
\hline & 11 Years and over & 27 & 58,00 & & & \\
\hline \multirow{5}{*}{$\begin{array}{l}\text { Authoritarian } \\
\text { Classroom } \\
\text { management } \\
\text { profile }\end{array}$} & 1 year and under & 15 & 59,30 & \multirow[t]{5}{*}{4} & \multirow[t]{5}{*}{6,084} & \multirow[t]{5}{*}{0,193} \\
\hline & 2-4 years & 40 & 43,10 & & & \\
\hline & $5-7$ years & 11 & 62,27 & & & \\
\hline & $8-10$ years & 7 & 52,14 & & & \\
\hline & 11 Years and over & 27 & 51,35 & & & \\
\hline \multirow{5}{*}{$\begin{array}{l}\text { Laissez-faire } \\
\text { Classroom } \\
\text { management } \\
\text { profile }\end{array}$} & 1 year and under & 15 & 49,87 & \multirow[t]{5}{*}{4} & \multirow[t]{5}{*}{2,266} & \multirow[t]{5}{*}{0,687} \\
\hline & 2-4 years & 40 & 48,34 & & & \\
\hline & 5-7 years & 11 & 55,82 & & & \\
\hline & $8-10$ years & 7 & 63,64 & & & \\
\hline & 11 Years and over & 27 & 48,48 & & & \\
\hline \multirow{5}{*}{$\begin{array}{c}\text { Total (General } \\
\text { scale) }\end{array}$} & 1 year and under & 15 & 47,97 & \multirow[t]{5}{*}{4} & \multirow[t]{5}{*}{2,309} & \multirow[t]{5}{*}{0,679} \\
\hline & 2-4 years & 40 & 48,24 & & & \\
\hline & 5-7 years & 11 & 57,14 & & & \\
\hline & $8-10$ years & 7 & 63,00 & & & \\
\hline & 11 Years and over & 27 & 49,31 & & & \\
\hline
\end{tabular}

There is no statistically significant difference between the total scores obtained from the Authoritative Classroom Management Profile sub-scale $\left(\chi^{2}(\mathrm{sd}=4, \mathrm{n}=100)=3,088 ; \mathrm{p}=0,543>0,05\right.$. There is no statistically significant difference between the total scores from the Sub-scale of Laissez-faire Classroom Management by occupation year $\left(\chi^{2}(\mathrm{sd}=4, \mathrm{n}=100)=6,084\right.$; $\mathrm{p}=0,193>0,05$. There is no statistically significant difference between the total scores obtained from the subscale of the Indifferent Class Management Profession by placement year $\left(\chi^{2}(s d=4, n=100)=2,266 ; p=0,687>0,05\right.$. There is no statistically significant difference between the total scores according to the placement year $\left(\chi^{2}(\mathrm{sd}=4, \mathrm{n}=100)=2,309 ; \mathrm{p}=0,679>0,0\right.$. 
The Levene Test was used to test the homogeneity of variances for the subscale of Authoritarian Classroom Management.

Table 7. Levene Test

\begin{tabular}{|c|c|c|c|c|}
\hline & Levene & $\begin{array}{c}\text { Degree of Freedom } \\
1\end{array}$ & $\begin{array}{c}\text { Degree of Freedom } \\
2\end{array}$ & $\mathrm{p}$ \\
\hline $\begin{array}{c}\text { Authoritarian Classroom management } \\
\text { profile }\end{array}$ & 0,109 & 4 & 95 & 0,979 \\
\hline
\end{tabular}

According to the results of the Levene test, for the Authoritarian Classroom Management Profile, $\mathrm{p}>0.05$, the variances are equal (homogenize). Here, parametric tests will be applied.

Table 8. Is there a difference in the secondary school teachers' Authoritarian sub-scale scores by placement year?

\begin{tabular}{|c|c|c|c|c|c|c|c|}
\hline & $\begin{array}{c}\text { Placement } \\
\text { year }\end{array}$ & $\begin{array}{c}\text { Source of } \\
\text { Variance }\end{array}$ & $\begin{array}{l}\text { Sum of } \\
\text { Square }\end{array}$ & $\begin{array}{c}\text { Degree of } \\
\text { Freedom }\end{array}$ & $\begin{array}{c}\text { Mean } \\
\text { Square }\end{array}$ & $\mathrm{F}$ & $\mathrm{p}$ \\
\hline \multirow{2}{*}{$\begin{array}{l}\text { Authoritarian } \\
\text { Classroom } \\
\text { Management } \\
\text { Profile }\end{array}$} & \multirow{2}{*}{$\begin{array}{l}1 \text { year and } \\
\text { under } \\
2-4 \text { years } \\
5-7 \text { years } \\
8-10 \text { years } \\
11 \text { Years } \\
\text { and over }\end{array}$} & $\begin{array}{c}\text { Gruplar } \\
\text { Aras1 }\end{array}$ & 15,393 & 4 & 3,848 & \multirow{2}{*}{1,337} & \multirow{2}{*}{0,262} \\
\hline & & Grup İçi & 273,517 & 95 & 2,879 & & \\
\hline
\end{tabular}

A statistically significant difference was not found when the scores obtained from the sub-scale of the Authoritarian Classroom Management Profile differed according to the placement $\operatorname{year}(\mathrm{F}(4,95)=1,337 ; \mathrm{p}=$ $0,262>0,05)$.

Table 9. Is there a difference in the secondary school teachers scale and subscale scores according to sex?

\begin{tabular}{|c|c|c|c|c|c|c|}
\hline & Sex & $\mathrm{N}$ & $\begin{array}{l}\text { Sum of } \\
\text { Square }\end{array}$ & $\begin{array}{l}\text { Order } \\
\text { Total } \\
\end{array}$ & M.W.U. & $\mathrm{p}$ \\
\hline \multirow{2}{*}{$\begin{array}{l}\text { Authoritarian } \\
\text { Classroom } \\
\text { Management } \\
\text { Profile }\end{array}$} & Women & 43 & 48,53 & 2087,00 & \multirow[t]{2}{*}{1141,000} & \multirow[t]{2}{*}{0,549} \\
\hline & Men & 57 & 51,98 & 2963,00 & & \\
\hline \multirow{2}{*}{$\begin{array}{l}\text { Authoritative } \\
\text { Classroom } \\
\text { management } \\
\text { profile }\end{array}$} & Women & 43 & 50,56 & 2174,00 & \multirow[t]{2}{*}{1223,000} & \multirow[t]{2}{*}{0,986} \\
\hline & Men & 57 & 50,46 & 2876,00 & & \\
\hline \multirow{2}{*}{$\begin{array}{l}\text { Laissez-faire } \\
\text { Classroom } \\
\text { management } \\
\text { profile }\end{array}$} & Women & 43 & 54,07 & 2325,00 & \multirow[t]{2}{*}{1072,000} & \multirow[t]{2}{*}{0,275} \\
\hline & Men & 57 & 47,81 & 2725,00 & & \\
\hline \multirow{2}{*}{$\begin{array}{c}\text { Indifferent } \\
\text { Classroom } \\
\text { management } \\
\text { profile }\end{array}$} & Women & 43 & 52,66 & 2264,50 & \multirow[t]{2}{*}{1132,500} & \multirow[t]{2}{*}{0,508} \\
\hline & Men & 57 & 48,87 & 2785,50 & & \\
\hline \multirow{2}{*}{ Total } & Women & 43 & 52,93 & 2276,00 & \multirow[t]{2}{*}{1121,000} & \multirow[t]{2}{*}{0,464} \\
\hline & Men & 57 & 48,67 & 2774,00 & & \\
\hline
\end{tabular}


From Table 9 above, a statistically significant difference was not observed when the scores of the Authoritarian Classroom Management Profile subscale differed according to the sex ( $U=1141,000 ; p=0,549>0,05)$. A statistically significant difference was not observed when the scores of the authoritative Classroom Management Profile subscale differed according to the sex $(U=1223,000 ; p=0,986>0,05)$. A statistically significant difference was not observed when the scores of the laissez-faire Classroom Management Profile subscale differed according to the sex ( $\mathrm{U}=1072,000$; $\mathrm{p}=0,275>0,05)$. A statistically significant difference was not observed when the scores of the Indifferent Classroom Management Profile subscale differed according to the sex $(U=1132,500 ; p=0,508>0,05)$. Also, there was no statistically significant difference according to the sexes whether total (overall scale) scores were different or not $(U=1121,000 ; p=0,464>0,05)$.

Table 10. Primary school teachers' Distribution by Placement Year

\begin{tabular}{|c|c|c|}
\hline & Frequency & Percent (\%) \\
\hline 1 year and under & 3 & 3,7 \\
\hline 2-4 years & 9 & 11,1 \\
\hline 5-7 years & 10 & 12,3 \\
\hline 8-10 years & 24 & 29,6 \\
\hline 11 Years and over & 35 & 43,2 \\
\hline Total & 81 & 100,0 \\
\hline
\end{tabular}

Table 11. Distribution of Primary school teachers by Sex

\begin{tabular}{|c|c|c|}
\hline & Frequency & Percent $(\%)$ \\
\hline Women & 37 & 45,7 \\
\hline Men & 44 & 54,3 \\
\hline Total & 81 & 100,0 \\
\hline
\end{tabular}

Table 12. Primary school teachers' Distribution by Age Groups

\begin{tabular}{|c|c|c|}
\hline & Frequency & Percent $(\%)$ \\
\hline 24 Age and under & 4 & 4,9 \\
\hline 25-29 Age aras1 & 14 & 17,3 \\
\hline 30-34 Age aras1 & 30 & 37,0 \\
\hline 35-39 Age aras1 & 14 & 17,3 \\
\hline 40 Age ve üzeri & 19 & 23,5 \\
\hline Total & 81 & 100,0 \\
\hline
\end{tabular}


Table 13. Is there a difference in primary school teachers' sum of Scale and the Subscales scores by Age?

\begin{tabular}{|c|c|c|c|c|c|c|}
\hline & Age & $\mathrm{n}$ & Order Average & $\begin{array}{l}\text { Degree of } \\
\text { Freedom }\end{array}$ & $\chi^{2}$ & $P$ \\
\hline \multirow{5}{*}{$\begin{array}{l}\text { Authoritarian } \\
\text { Classroom } \\
\text { Management } \\
\text { Profile }\end{array}$} & $\begin{array}{l}24 \text { Age and } \\
\text { under }\end{array}$ & 4 & 44,75 & \multirow[t]{5}{*}{4} & \multirow[t]{5}{*}{0,804} & \multirow[t]{5}{*}{0,938} \\
\hline & $\begin{array}{c}\text { 25-29 Age } \\
\text { aras1 }\end{array}$ & 14 & 38,04 & & & \\
\hline & $\begin{array}{c}\text { 30-34 Age } \\
\text { aras1 }\end{array}$ & 30 & 43,12 & & & \\
\hline & $\begin{array}{c}\text { 35-39 Age } \\
\text { aras1 }\end{array}$ & 14 & 38,21 & & & \\
\hline & $\begin{array}{c}40 \text { Age ve } \\
\text { üzeri }\end{array}$ & 19 & 41,11 & & & \\
\hline \multirow{5}{*}{$\begin{array}{l}\text { Authoritative } \\
\text { Classroom } \\
\text { management } \\
\text { profile }\end{array}$} & $\begin{array}{c}24 \text { Age and } \\
\text { under }\end{array}$ & 4 & 34,38 & \multirow[t]{5}{*}{4} & \multirow[t]{5}{*}{0,874} & \multirow[t]{5}{*}{0,928} \\
\hline & $\begin{array}{c}\text { 25-29 Age } \\
\text { aras1 }\end{array}$ & 14 & 40,61 & & & \\
\hline & $\begin{array}{c}\text { 30-34 Age } \\
\text { aras1 }\end{array}$ & 30 & 40,32 & & & \\
\hline & $\begin{array}{c}\text { 35-39 Age } \\
\text { aras1 }\end{array}$ & 14 & 39,82 & & & \\
\hline & $\begin{array}{c}40 \text { Age ve } \\
\text { üzeri }\end{array}$ & 19 & 44,63 & & & \\
\hline \multirow{5}{*}{$\begin{array}{c}\text { Indifferent } \\
\text { Classroom } \\
\text { management } \\
\text { profile }\end{array}$} & $\begin{array}{c}24 \text { Age and } \\
\text { under }\end{array}$ & 4 & 52,25 & \multirow[t]{5}{*}{4} & \multirow[t]{5}{*}{5,180} & \multirow[t]{5}{*}{0,269} \\
\hline & $\begin{array}{c}\text { 25-29 Age } \\
\text { aras1 }\end{array}$ & 14 & 49,21 & & & \\
\hline & $\begin{array}{c}\text { 30-34 Age } \\
\text { aras1 }\end{array}$ & 30 & 37,28 & & & \\
\hline & $\begin{array}{c}\text { 35-39 Age } \\
\text { aras1 }\end{array}$ & 14 & 45,43 & & & \\
\hline & $\begin{array}{c}40 \text { Age ve } \\
\text { üzeri }\end{array}$ & 19 & 35,18 & & & \\
\hline
\end{tabular}

There is no statistically significant difference in the total scores from the subscale of the Authoritarian Classroom Management Profile by age $\left(\chi^{2}(s d=4, n=81)=0,804 ; p=0,938>0,05\right.$. There is no statistically significant difference in the total scores from the subscale of the Authoritative Classroom Management Profile by age $\left(\chi^{2}(\mathrm{sd}=4, \quad \mathrm{n}=81)=0,874\right.$; $\mathrm{p}=0,928>0,05$. There is no statistically significant difference in the total scores from the subscale of the Indifferent Classroom Management Profile by age $\left(\chi^{2}(s d=4, n=81)=5,180 ; p=0,269>0,05\right.$. The Levene Test was used to test the homogeneity of variances for the Total and Leissez-faire Class Management Profiles. 
Table 14. Levene Test

\begin{tabular}{|c|c|c|c|c|}
\hline & Levene & $\frac{\text { Degree of }}{\text { Freedom 1 }}$ & $\begin{array}{c}\text { Degree of } \\
\text { Freedom 2 }\end{array}$ & $\mathrm{p}$ \\
\hline $\begin{array}{c}\text { Authoritarian Classroom } \\
\text { management profile }\end{array}$ & 0,671 & 4 & 76 & 0,614 \\
\hline Total & 1,775 & 4 & 76 & 0,143 \\
\hline
\end{tabular}

According to the results of the Levene test, for Leissez faire Classroom Management Profiles and Total $p>0.05$, the variances are equal (homogenize). Parametric tests will be applied.

Table 15. Is there a difference in Primary school teachers Authoritarian Classroom Management Profiles Subscale Score by Age?

\begin{tabular}{|c|c|c|c|c|c|c|c|}
\hline & Age & $\begin{array}{l}\text { Source of } \\
\text { variation }\end{array}$ & $\begin{array}{l}\text { Sum of } \\
\text { squares }\end{array}$ & $\begin{array}{l}\text { Degree of } \\
\text { Freedom }\end{array}$ & $\begin{array}{l}\text { Relatives } \\
\text { of squares }\end{array}$ & $\mathrm{F}$ & $\mathrm{p}$ \\
\hline \multirow{2}{*}{$\begin{array}{l}\text { Authoritarian } \\
\text { Classroom } \\
\text { management } \\
\text { profile }\end{array}$} & $\begin{array}{c}24 \text { Age } \\
\text { and } \\
\text { under } \\
25-29 \\
\text { Age } \\
30-34\end{array}$ & $\begin{array}{c}\text { Between- } \\
\text { subjects }\end{array}$ & 22,939 & 4 & 5,735 & \multirow[b]{2}{*}{1,591} & \multirow[b]{2}{*}{0,185} \\
\hline & $\begin{array}{c}\text { Age } \\
35-39 \\
\text { Age } \\
40 \text { Age } \\
\text { and } \\
\text { over }\end{array}$ & $\begin{array}{l}\text { Within- } \\
\text { group }\end{array}$ & 273,950 & 76 & 3,605 & & \\
\hline \multirow[b]{2}{*}{ Total } & $\begin{array}{c}24 \text { Age } \\
\text { and } \\
\text { under } \\
25-29 \\
\text { Age } \\
30-34\end{array}$ & $\begin{array}{l}\text { Between- } \\
\text { subjects }\end{array}$ & 6,251 & 4 & 1,563 & \multirow[b]{2}{*}{0,110} & \multirow[b]{2}{*}{0,979} \\
\hline & $\begin{array}{c}\text { Age } \\
35-39 \\
\text { Age } \\
40 \text { Age } \\
\text { and } \\
\text { over }\end{array}$ & $\begin{array}{l}\text { Within- } \\
\text { group }\end{array}$ & 1084,070 & 76 & 14,264 & & \\
\hline
\end{tabular}

A statistically significant difference was not observed when the scores from the Leissez faire Classroom Management Profile subscale differed according to age $(\mathrm{F}(, 76)=1,591 ; \mathrm{p}=0,185>0,05)$. There was no statistically significant difference when comparing the scores obtained from the total (overall scale) according to age $(\mathrm{F}(4,76)=0,110 ; \mathrm{p}=0,979>0,05)$.

The Levene Test was used to test the homogeneity of variances for the Authoritative Classroom Management, the Leissez faire Classroom Management Profile subscale. 
Table 16. Levene Test

\begin{tabular}{|c|c|c|c|c|}
\hline & Levene & $\frac{\text { Degree of Freedom }}{1}$ & Degree of Freedom & $\mathrm{p}$ \\
\hline $\begin{array}{c}\text { Authoritative Classroom } \\
\text { management }\end{array}$ & 2,442 & 3 & 77 & 0,071 \\
\hline $\begin{array}{c}\text { Laissez-faire Classroom } \\
\text { management }\end{array}$ & 0,892 & 3 & 77 & 0,449 \\
\hline
\end{tabular}

According to the results of the Levene test, the admissible class management profile equals (homogenises) the variances because $p>0,05$ for the authoritative and Leissez faire Class Management Profile. Parametric tests will be applied.

Table 17. Is there a difference in Primary school teachers' sum of Scale and the Subscales scores by placement years?

\begin{tabular}{|c|c|c|c|c|c|c|}
\hline & $\begin{array}{l}\text { Placement } \\
\text { years }\end{array}$ & $\mathrm{N}$ & $\begin{array}{c}\text { Order } \\
\text { Average }\end{array}$ & $\begin{array}{c}\text { Degree of } \\
\text { freedom }\end{array}$ & $\chi^{2}$ & $\mathrm{P}$ \\
\hline \multirow{4}{*}{$\begin{array}{l}\text { Authoritarian } \\
\text { Classroom } \\
\text { Management } \\
\text { Profile }\end{array}$} & $\begin{array}{c}4 \text { years and } \\
\text { under }\end{array}$ & 12 & 39,96 & \multirow[t]{4}{*}{3} & \multirow[t]{4}{*}{3,557} & \multirow[t]{4}{*}{0,313} \\
\hline & $5-7$ years & 10 & 29,50 & & & \\
\hline & $8-10$ years & 24 & 45,69 & & & \\
\hline & $\begin{array}{c}11 \text { Years and } \\
\text { over }\end{array}$ & 35 & 41,43 & & & \\
\hline \multirow{4}{*}{$\begin{array}{c}\text { Indifferent } \\
\text { Classroom } \\
\text { Management } \\
\text { Profile }\end{array}$} & $\begin{array}{c}4 \text { years and } \\
\text { under }\end{array}$ & 12 & 53,67 & \multirow[t]{4}{*}{3} & \multirow[t]{4}{*}{4,759} & \multirow[t]{4}{*}{0,190} \\
\hline & 5-7 years & 10 & 38,60 & & & \\
\hline & $8-10$ years & 24 & 36,12 & & & \\
\hline & $\begin{array}{c}11 \text { Years and } \\
\text { over }\end{array}$ & 35 & 40,69 & & & \\
\hline \multirow{4}{*}{$\begin{array}{c}\text { Total (General } \\
\text { scale) }\end{array}$} & $\begin{array}{c}4 \text { years and } \\
\text { under }\end{array}$ & 12 & 38,08 & \multirow[t]{4}{*}{3} & \multirow[t]{4}{*}{2,214} & \multirow[t]{4}{*}{0,529} \\
\hline & $5-7$ years & 10 & 42,85 & & & \\
\hline & $8-10$ years & 24 & 36,12 & & & \\
\hline & $\begin{array}{c}11 \text { Years and } \\
\text { over }\end{array}$ & 35 & 44,81 & & & \\
\hline \multirow{4}{*}{$\begin{array}{l}\text { Authoritative } \\
\text { Classroom } \\
\text { management } \\
\text { profile }\end{array}$} & $\begin{array}{c}4 \text { years and } \\
\text { under }\end{array}$ & 12 & 39,96 & \multirow[t]{4}{*}{3} & \multirow[t]{4}{*}{3,557} & \multirow[t]{4}{*}{0,313} \\
\hline & 5-7 years & 10 & 29,50 & & & \\
\hline & $8-10$ years & 24 & 45,69 & & & \\
\hline & $\begin{array}{l}11 \text { Years } \\
\text { and over }\end{array}$ & 35 & 41,43 & & & \\
\hline \multirow{4}{*}{$\begin{array}{l}\text { Laissez-faire } \\
\text { Classroom } \\
\text { management } \\
\text { profile }\end{array}$} & $\begin{array}{c}4 \text { years and } \\
\text { under }\end{array}$ & 12 & 53,67 & \multirow[t]{4}{*}{3} & \multirow[t]{4}{*}{4,759} & \multirow[t]{4}{*}{0,190} \\
\hline & $5-7$ years & 10 & 38,60 & & & \\
\hline & $8-10$ years & 24 & 36,12 & & & \\
\hline & $\begin{array}{l}11 \text { Years } \\
\text { and over }\end{array}$ & 35 & 40,69 & & & \\
\hline Total (General & 4 year and & 12 & 38,08 & 3 & 2,214 & 0,529 \\
\hline
\end{tabular}




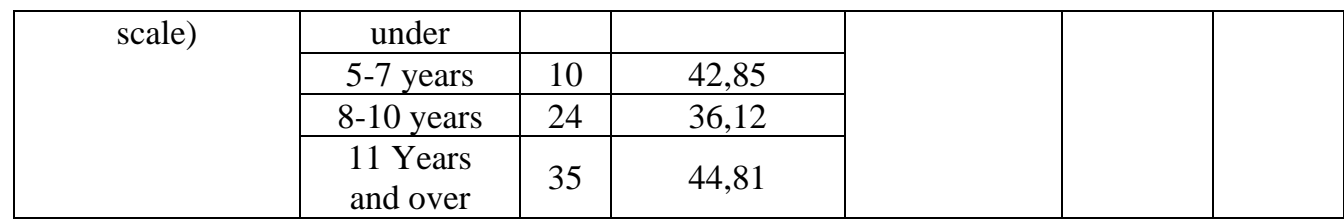

A statistically significant difference was not observed when the scores obtained from the Authoritarian, Indifferent, and Authoritative Classroom Management Profile subscale differed according to the placement year. A statistically significant difference was observed when the scores obtained from the Leissez faire Classroom Management Profile subscale differed according to the placement year $(\mathrm{F}(3,77)=4,205 ; \mathrm{p}=0,008<0,05)$. Post-hoc test statistics were applied to determine the source of significant difference between the groups as a result of this analysis. The average of 4 years and six groups $(9,4167 \pm 1,24011)$ is lower than that of 5-7 years $(11,3000 \pm 1,41814)$. The average of 4 years and six groups $(9,4167 \pm$ $1,24011)$ is lower than 11 years and over group $(10,9143 \pm 2,06328)$. The average of the group of 5-7 years $(11,3000 \pm 1,41814)$ is higher than the group of $8-10$ years $(9,6667 \pm 1,80980)$. The average of the group of $8-10$ years $(9,6667 \pm 1,80980)$ is lower than that of the group of 11 years and over $(10,9143 \pm 2,06328)$.

The Levene Test was used to test the homogeneity of the variances for the total (General Scale).

Table 18. Levene Test

\begin{tabular}{|c|c|c|}
\hline & \multicolumn{2}{|c|}{ Levene Test } \\
\hline & $\mathrm{F}$ & $\mathrm{p}$ \\
\hline Total (General scale) & 0,050 & 0,824 \\
\hline
\end{tabular}

Equal variances (homogeneity) because $\mathrm{p}>0.05$ for total (General Scale). Parametric tests will be applied.

Table 19. Is there a difference in Primary school teachers' Classroom Management Subscales Scores by sex?

\begin{tabular}{|c|c|c|c|c|c|c|}
\hline & Sex & $\mathrm{n}$ & $\begin{array}{c}\text { Order } \\
\text { Average }\end{array}$ & $\begin{array}{l}\text { Order } \\
\text { Total }\end{array}$ & M.W.U. & $\mathrm{p}$ \\
\hline \multirow{2}{*}{$\begin{array}{c}\text { Authoritarian } \\
\text { Classroom } \\
\text { Management Profile }\end{array}$} & Women & 37 & 43,77 & 1619,50 & \multirow[t]{2}{*}{711,500} & \multirow[t]{2}{*}{0,319} \\
\hline & Men & 44 & 38,67 & 1701,50 & & \\
\hline \multirow{2}{*}{$\begin{array}{c}\text { Authoritative } \\
\text { Classroom } \\
\text { management profile }\end{array}$} & Women & 37 & 41,64 & 1540,50 & \multirow[t]{2}{*}{790,500} & \multirow[t]{2}{*}{0,820} \\
\hline & Men & 44 & 40,47 & 1780,50 & & \\
\hline \multirow{2}{*}{$\begin{array}{c}\text { Laissez-faire } \\
\text { Classroom } \\
\text { management profile }\end{array}$} & Women & 37 & 37,03 & 1370,00 & \multirow[t]{2}{*}{667,000} & \multirow[t]{2}{*}{0,157} \\
\hline & Men & 44 & 44,34 & 1951,00 & & \\
\hline \multirow{2}{*}{$\begin{array}{l}\text { Indifferent Classroom } \\
\text { management profile }\end{array}$} & Women & 37 & 40,04 & 1481,50 & \multirow[t]{2}{*}{778,500} & \multirow[t]{2}{*}{0,733} \\
\hline & Men & 44 & 41,81 & 1839,50 & & \\
\hline
\end{tabular}


A statistically significant difference was not observed when the scores of the Authoritarian Classroom Management Profile subscale differed according to the sex $(U=711,500 ; p=0,319<0,05)$. A statistically significant difference was not observed when the scores of the authoritative Classroom Management Profile subscale differed according to the sex ( $U=790,500$; $\mathrm{p}=0,820>0,05)$. A statistically significant difference was not observed when the scores of the laissez-faire Classroom Management Profile subscale differed according to the sex $(U=667,000 ; p=0,157<0,05)$. A statistically significant difference was not observed when the scores of the Indifferent Classroom Management Profile subscale differed according to the sex $(\mathrm{U}=$ 778,500; $\mathrm{p}=0,733>0,05)$.

Table 20. Is there a difference in Primary school teachers' Sum of Scale Scores Differ by Sex?

\begin{tabular}{|c|c|c|c|c|c|c|c|}
\hline & Sex & $\mathrm{N}$ & Mean & $\begin{array}{c}\text { Standard } \\
\text { deviation }\end{array}$ & $\mathrm{t}$ & $\begin{array}{c}\text { Degree of } \\
\text { Freedom }\end{array}$ & $\mathrm{p}$ \\
\hline \multirow{2}{*}{ Total } & Women & 37 & 40,0811 & 3,89000 & \multirow{2}{*}{$-0,589$} & 79 & 0,557 \\
\cline { 2 - 8 } & Men & 44 & 40,5682 & 3,54636 & 79 & \\
\hline
\end{tabular}

There was no statistically significant difference between the scores obtained from the total scale according to the sexes $\left(\mathrm{t}_{(79)}=-0,589 ; \mathrm{p}=\right.$ $0,557>0,05)$.

Table 21. Is there a difference in sum of Scale and Subscales scores by Branch?

\begin{tabular}{|c|c|c|c|c|c|c|}
\hline & Branch & $\mathrm{n}$ & $\begin{array}{c}\text { Order } \\
\text { Average }\end{array}$ & $\begin{array}{l}\text { Order } \\
\text { Total }\end{array}$ & M.W.U. & $\mathrm{p}$ \\
\hline \multirow{2}{*}{$\begin{array}{l}\text { Authoritarian } \\
\text { Classroom } \\
\text { Management } \\
\text { Profile }\end{array}$} & Secondary School & 100 & 91,31 & 9131,00 & \multirow[t]{2}{*}{4019,000} & \multirow[t]{2}{*}{0,928} \\
\hline & $\begin{array}{l}\text { Primary school } \\
\text { teacher }\end{array}$ & 81 & 90,62 & 7340,00 & & \\
\hline \multirow{2}{*}{$\begin{array}{l}\text { Authoritative } \\
\text { Classroom } \\
\text { management } \\
\text { profile }\end{array}$} & Secondary School & 100 & 92,56 & 9256,50 & \multirow[t]{2}{*}{3893,500} & \multirow[t]{2}{*}{0,649} \\
\hline & $\begin{array}{l}\text { Primary school } \\
\text { teacher }\end{array}$ & 81 & 89,07 & 7214,50 & & \\
\hline \multirow{2}{*}{$\begin{array}{l}\text { Laissez-faire } \\
\text { Classroom } \\
\text { management } \\
\text { profile }\end{array}$} & Secondary School & 100 & 91,15 & 9115,00 & \multirow[t]{2}{*}{4035,000} & \multirow[t]{2}{*}{0,965} \\
\hline & $\begin{array}{l}\text { Primary school } \\
\text { teacher }\end{array}$ & 81 & 90,81 & 7356,00 & & \\
\hline \multirow{2}{*}{$\begin{array}{l}\text { Indifferent } \\
\text { Classroom } \\
\text { management } \\
\text { profile }\end{array}$} & Secondary School & 100 & 95,80 & 9580,50 & \multirow[t]{2}{*}{3569,500} & \multirow[t]{2}{*}{0,163} \\
\hline & $\begin{array}{l}\text { Primary school } \\
\text { teacher }\end{array}$ & 81 & 85,07 & 6890,50 & & \\
\hline \multirow[b]{2}{*}{ Total } & Secondary School & 100 & 95,80 & 9579,50 & \multirow[t]{2}{*}{3570,500} & \multirow[t]{2}{*}{0,169} \\
\hline & $\begin{array}{l}\text { Primary school } \\
\text { teacher }\end{array}$ & 81 & 85,08 & 6891,50 & & \\
\hline
\end{tabular}

A statistically significant difference was not observed when the scores obtained from the subscale of the Authoritarian Classroom 
Management Profile differed ( $U=4019,000 ; p=0,928>0,05)$. A statistically significant difference was not observed when the scores obtained from the subscale of the Authoritative Classroom Management Profile differed ( $\mathrm{U}=$ $3893,500 ; \mathrm{p}=0,649>0,05)$. A statistically significant difference was not observed when the scores obtained from the subscale of the Laissez-faire Classroom Management Profile differed ( $U=4035,000 ; p=0,965>0,05)$. A statistically significant difference was not observed when the scores obtained from the subscale of the Indifferent Classroom Management Profile differed $(\mathrm{U}=3569,500 ; \mathrm{p}=0,163>0,05)$. There was no statistically significant difference when the total scores (general scale) were different according to the field $(U=3570,500 ; p=0,169>0,05)$.

\section{Conclusion}

No statistically significant difference was found between the scores obtained from the general and sub-dimensions of the classroom management profile inventory, especially when primary school teachers and secondary school teachers were compared. Neither the secondary school nor primary school teachers' scores on the sub-dimensions and sums of the classroom management profile inventory differ according to branch, age, and sex. However, it was found that the primary school teachers differ in the profiles according to the placement year. In this sub-dimension, the lowest score belongs to the group "4 years and below", while the highest score belongs to the group "5-7 years". Besides, there is no statistically significant difference on secondary school teachers' scores on the sub-dimensions and sums of the classroom management profile inventory according to placement year.

When the relevant literature is examined, Ekici (2004) found that first level education classroom teacher mostly preferred the authoritative classroom management profile. Furthermore, a statistically significant difference was found at the 0.05 confidence level among some of the classroom management profiles in terms of teachers' sex, professional seniority, socioeconomic condition of the schools they work, and student numbers in the classroom.

In another research, Y1lmaz (2011) found that more than half of the primary school teachers have the "Authoritative Classroom Management Style". This style however involves laissez-faire, authoritarian, and indifferent classroom management styles. In addition, teachers have a high level agreement to each classroom management style. Generally, authoritative classroom management styles are preferred by students. According to a research on class management profiles, Çiftçi (2015) stated that female teachers are more authoritarian than male teachers, teachers having 6-10 years seniority, widowed teachers having 26-30 and 31above 31 years seniority. Consequently, widowed teachers produce more 
adrift class management profile than single teachers. Also, teachers between 26-30 years old perform more adrift class management profile compared to teachers between 31-40 years old.

According to Erdoğan and Kurt's (2015) review research, the findings indicated that teachers' ability to create this interactive classroom environment was influenced by some variables such as teacher beliefs and teachers' expectations of the students. Also, it demonstrated the complex nature of classroom management as it was investigated through a rich set of variables in the study. Furthermore, it provided implications for teachers and teacher trainers on classroom management. Finally, this study outlines the critical areas of research on classroom management and identifies areas for further research.

\section{References:}

1. Balay, R. (2012). Sinıf Yönetimi. Ankara: Pegem.

2. Brophy, J. E. (1996). Enhancing students' socialization: Key elements. ERIC Clearinghouse on Elementary and Early Childhood Education, University of Illinois.

3. Çiftçi, A. S. (2015). İlkokul ögrretmenlerinin sınıf yönetim tarzlarl ve demokratik değerlere ilişkin görüşleri arasındaki ilişki (Master's thesis, Çanakkale Onsekiz Mart Üniversitesi Eğitim Bilimleri Enstitüsü).

4. Dunbar, C. (2004). Best practices in classroom management. Michigan State University, 1-32.

5. Ekici, G. (2004). İlköğretim I. kademe öğretmenlerinin sinıf yönetimi profillerinin değerlendirilmesi. Eğitim ve Bilim, 29(131).

6. Erdogan, M., \& Kurt, A. (2015). A review of research on classroom management in Turkey. Procedia-Social and Behavioral Sciences, 186, 9-14.

7. Hue, M. T., \& Li, W. S. (2008). Classroom management: Creating a positive learning environment (Vol. 1). Hong kong University Press.

8. Karasar, N. (2014). Bilimsel Araştırma Yöntemi. Ankara: Nobel.

9. Marzano, R. J., Marzano, J. S., \& Pickering, D. (2003). Classroom management that works: Research-based strategies for every teacher. ASCD.

10. Terzi, A. R. (2002). Sınıf yönetimi açısından etkili öğretmen davranışları. Milli Eğitim Dergisi, (155-156), 155-156.

11. Yılmaz, K. (2011). İlköğretim Okulu Öğretmenlerinin Sınıf Yönetimi Tarzları ile Demokratik Değerlere İlişkin Görüşleri Arasındaki İlişki. Journal of Values Education, 9(21).

12. Yisrael, S. B. (2012). Classroom management: A guide for urban school teachers. R\&L Education. 